\title{
How to make a fragile network robust and vice versa
}

\author{
André A. Moreira,, , $*$ José S. Andrade Jr., ${ }^{1,2}$ Hans J. Herrmann, ${ }^{1,2}$ and Joseph O. Indekeu ${ }^{3}$ \\ ${ }^{1}$ Departamento de Fúsica, Universidade Federal do Ceará, 60451-970 Fortaleza, Ceará, Brazil \\ ${ }^{2}$ Computational Physics, IfB, ETH-Hönggerberg, Schafmattstrasse 6, 8093 Zürich, Switzerland \\ ${ }^{3}$ Instituut voor Theoretische Fysica, Katholieke Universiteit Leuven, B-3001 Leuven, Belgium
}

(Dated: November 9, 2018)

\begin{abstract}
We investigate topologically biased failure in scale-free networks with degree distribution $P(k) \propto$ $k^{-\gamma}$. The probability $p$ that an edge remains intact is assumed to depend on the degree $k$ of adjacent nodes $i$ and $j$ through $p_{i j} \propto\left(k_{i} k_{j}\right)^{-\alpha}$. By varying the exponent $\alpha$, we interpolate between random $(\alpha=0)$ and systematic failure. For $\alpha>0(<0)$ the most (least) connected nodes are depreciated first. This topological bias introduces a characteristic scale in $P(k)$ of the depreciated network, marking a crossover between two distinct power laws. The critical percolation threshold, at which global connectivity is lost, depends both on $\gamma$ and on $\alpha$. As a consequence, network robustness or fragility can be controlled through fine tuning of the topological bias in the failure process.
\end{abstract}

PACS numbers: 64.60.aq, 89.75.Hc 64.60.ah,

Scale-free networks, with power-law degree distribution $P(k) \propto k^{-\gamma}$, are remarkably resistant to random failure [1, 2]. This quality is important when failure is to be avoided, as in the air-transportation network. It has been speculated that, also in nature, scale-free design evolves as a way to achieve robustness [3]. On the other hand, robustness may be a problem when one tries to halt an epidemic. The fundamental question we ask and answer in this work is how one can delicately control whether a network is fragile or robust.

Previous work has mostly concentrated on homogeneous networks, in which all edges have the same chance to fail. However, by design or evolution the most critical edges of the network may become less prone to failure. Also, a targeted attack can disrupt the network after only a small fraction of edges fail [1, 4]. This shows that in heterogeneous networks the topology alone does not determine the susceptibility of breakdown.

The critical properties of static phenomena and dynamical processes are affected by the topology of the network of interactions [5]. It was recently shown [6, 7] that, by accounting for a topology dependence in the interaction strength between the nodes, $J_{i j} \propto\left(k_{i} k_{j}\right)^{-\alpha}$, one obtains a critical behavior that mimics the case of homogeneous interaction but with a different degree distribution. The system with exponent $\gamma$ and topology-dependent interactions can be mapped to a homogeneous one, $\alpha=0$, but with an effective exponent $\gamma^{\prime}$, given by [,, 7$]$

$$
\gamma^{\prime}=\frac{\gamma-\alpha}{1-\alpha}
$$

We focus on failure in scale-free complex networks, mediated by a dynamical process that depends on the network topology. Disregarding the presence of correlations, any such dependence has to be related only to the node

*Electronic address: auto@fisica.ufc.br degree $k$. We have to choose between two possible approaches, namely failure of the nodes or, as we implement here, failure of the edges. We express the failure probability for an edge between nodes $i$ and $j$ as $q_{i j}=q_{i j}\left(k_{i}, k_{j}\right)$. We assume that the network depreciation occurs through a probability of occupation of an edge $p_{i j}=1-q_{i j}$, which depends on the degrees of the vertices,

$$
p_{i j} \propto w_{i j}=\left(k_{i} k_{j}\right)^{-\alpha},
$$

where $w_{i j}$ is the topology-dependent weight of the edge. Equation (2) is in the same spirit as the degree-dependent interaction proposed in [6, 7]. Since failure can be related to the purely geometrical model of percolation, its understanding does not require interactions but can be achieved directly in terms of topological properties.

A topology-dependent depreciation allows to interpolate smoothly between random failure $(\alpha=0)$ and intentional attack of links between hubs $(\alpha>0)$, or intentional depreciation of edges between the least connected nodes $(\alpha<0)$. We shall see that $\alpha \in[2-\gamma, 1]$ defines the useful range of topological bias in the context of scalefree networks with finite mean degree $(\gamma>2)$. Degreedependent failure was also studied in [8] for the case of node removal, while edge removal was investigated in [9].

For uncorrelated networks, homogeneous random failure $(\alpha=0)$ can be solved using a mean-field approach [2, 10, 11, 12]. Close to the critical fraction of occupied edges $f_{c}$, the size of the largest connected cluster grows as $\left(f-f_{c}\right)^{\beta}$, where the critical exponent $\beta$ depends on $\gamma[12]$. For $2<\gamma \leq 3$ the critical point vanishes, $f_{c} \rightarrow 0$. As a consequence, we may say that networks with $\gamma \leq 3$ are robust while networks with $\gamma>3$ are fragile. All these results, however, are only relevant for the case of random failure. Henceforth, we will call the regime $\alpha>0$ "centrally biased" $(\mathbf{C B})$. The converse regime, $\alpha<0$, will be termed "peripherally biased" (PB).

To build our scale-free networks, we use the configuration model (CM) [11]. The parameters of this model are the exponent $\gamma$, the number of nodes (or vertices) $N_{v}$, 


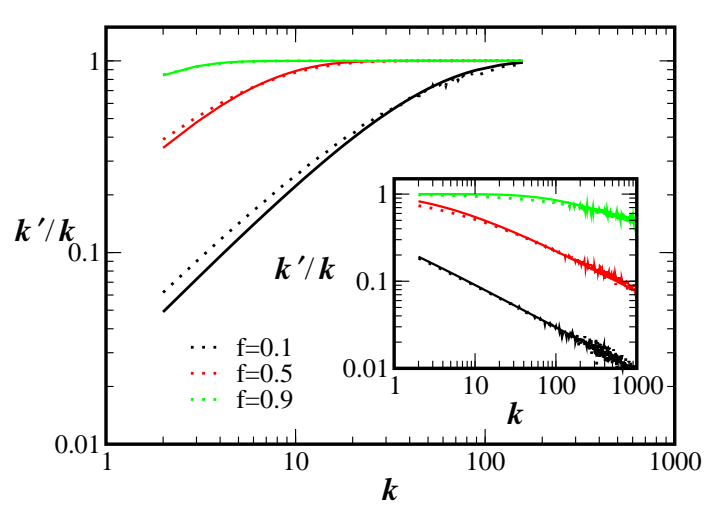

FIG. 1: Average degree reduction in the depreciated network as a function of the original node degree. The main panel shows results for networks with $\gamma=4$ submitted to PB with $\alpha=-1$. One can see that the fraction $k^{\prime} / k$ grows as a power law with degree $k$, saturating at 1 at a scale that depends on the fraction $f=n / N_{e}$ of edges in the depreciated network. The dotted lines are the numerical results obtained from 10 network realizations of size $N_{v}=10^{5}$. For each one of these networks, the depreciation has been applied 10 times. The continuous lines are the best fit to the data of Eq. (4) with the free parameter $C(f)=0.025,0.22$ and 0.92 for $f=0.1,0.3$ and 0.9 , respectively. The inset shows the same as in the main panel, but for a network with $\gamma=2.5$ subjected to $\mathrm{CB}$ with $\alpha=0.5$. In this case, the fraction $k^{\prime} / k$ decays with $k$. For small scales one may find a saturation depending on the fraction of edges in the depreciated network. The continuous lines are the best fits to the data of Eq. (4) with values $C(f)=$ $0.28,2.5$ and 19 for $f=0.1,0.3$ and 0.9 , respectively.

and the minimum degree allowed $k_{\min }$. Unless said otherwise, all the networks studied in this work have $k_{\min }=2$. Depending on these parameters and on the particular realization, we obtain a different number of edges $N_{e}$. In this model, the degrees of the nodes are determined initially from the desired distribution and then connections are assigned at random.

To study the depreciation process, we start from a total failure scenario, i.e., with all edges being initially removed from the network. We then gradually include the edges back, with probability proportional to some weight $w_{i j}$ that we will assume to follow Eq. (2). By stopping this process at intermediate steps, we can obtain results for the percolation problem as the fraction of occupied edges grows from zero to one.

We now determine the probability $P_{i j}(f)$ that a particular edge connecting nodes $i$ and $j$ is present in the network after a fraction $f$ of the edges has already been included. This probability can be identified as $P_{i j}(f)=$ $1-\prod_{t=1}^{n}\left(1-w_{i j} / Z_{t}\right)$, where $Z_{t}$ is the mean (over the inclusion process) of the sum of weights of all edges that have not yet been included in the network at step $t$, and $n=f N_{e}$ is the number of included edges. Assuming $w_{i j} \ll Z_{t}$, we can write,

$$
P_{i j}(f) \approx 1-e^{-D(f)\left(k_{i} k_{j}\right)^{-\alpha}},
$$

where the parameter $D(f)$ can be determined using $\sum P_{i j}=n . \quad$ Using $w_{i j}$ as defined in Eq. (2), the Kasteleyn-Fortuin construction [13] allows us to draw a parallel between the probability $P_{i j}$ and the degreedependent interaction previously proposed in [6, 7].

We now define $\rho_{k}(f)$ as the mean probability that an edge from a node with degree $k$ is present in the depreciated network. We then have to average $P_{i j}$ over the nearest neighbors of a node with degree $k, \rho_{k}=$ $\int_{k_{\min }}^{\infty} P_{n}\left(k_{n}\right)\left(1-\exp \left[-D(f)\left(k k_{n}\right)^{-\alpha}\right]\right) d k_{n}$. For uncorrelated networks the degree distribution of a neighbor is given by $P_{n}\left(k_{n}\right)=P\left(k_{n}\right) k_{n} /\langle k\rangle$. Performing the integration and examining the asymptotic behavior of the resulting incomplete Gamma function, we find that $\rho_{k}$ is well approximated by

$$
\rho_{k} \approx 1-e^{-C(f) k^{-\alpha}}
$$

with $C(f)=\frac{k_{\text {min }}^{-\alpha}(\gamma-2)}{\gamma-2+\alpha} D(f)$, provided $\alpha \in[2-\gamma, 1]$. The same range of $\alpha$ is also featured in previous work on networks with degree-dependent interactions [6, 7]. Equation (4) is confirmed by the numerical results shown in Fig. 1.

Equation. (4) can be used to determine the average degree of a node after depreciation, $k^{\prime}(k)=k \rho_{k}$. From that we can obtain the degree distribution of the depreciated network: $P^{\prime}\left(k^{\prime}\right)=k^{\prime \gamma}$ for $C(f) k^{-\alpha} \gg 1$, and $P^{\prime}\left(k^{\prime}\right)=k^{\prime \gamma^{\prime}}$ for $C(f) k^{-\alpha} \ll 1$, where $\gamma^{\prime}$ is given by Eq. (1). We find that the degree distribution after depreciation exhibits a crossover at a scale given by $k_{s} \propto C(f)^{1 / \alpha}$. As expected, the crossover is not present in the random failure case, $\alpha=0$. However, if the failure process is affected by the topological properties of the network, as modeled by Eq. (21), we have a characteristic scale $k_{s}$ that has not been observed before. The presence of this crossover is supported by the numerical results shown in Fig. 2. It is interesting to note that whether $\gamma$ or $\gamma^{\prime}$ controls the decay at large degree depends on the sign of $\alpha$. If $\alpha>0$ (CB), we have $\gamma<\gamma^{\prime}$ and $\gamma^{\prime}$ controls the decay at large degree, while for $\alpha<0$ (PB) the larger exponent, $\gamma$, is the controlling one. Thus, the largest of the two exponents $\gamma$ and $\gamma^{\prime}$ controls the asymptotic decay. A robust network with $\gamma \leq 3$ under CB and a fragile network with $\gamma>3$ under $\mathrm{PB}$ may result in networks with similar degree distributions after depreciation. The numerical results shown in Fig. 2 correspond to the degree distributions of networks under $\mathrm{CB}$ and $\mathrm{PB}$ failure.

Next we investigate the critical behavior associated with percolation. A network is above the critical point when a node connected to another node in the spanning cluster has on average at least one other connection, thus assuring that the cluster does not fragment. For an uncorrelated network, this condition is equivalent to $\left\langle k^{\prime 2}\right\rangle /\left\langle k^{\prime}\right\rangle>2$ [2]. In order to determine the critical fraction $f_{c}$ we perform the depreciation process until this critical condition is reached. In the simplest case where $\alpha=0$, if $\gamma>3$, the ratio $\left\langle k^{\prime 2}\right\rangle /\left\langle k^{\prime}\right\rangle$ converges to a finite value as $N_{v} \rightarrow \infty$. In this case $f_{c}>0$, characterizing a 


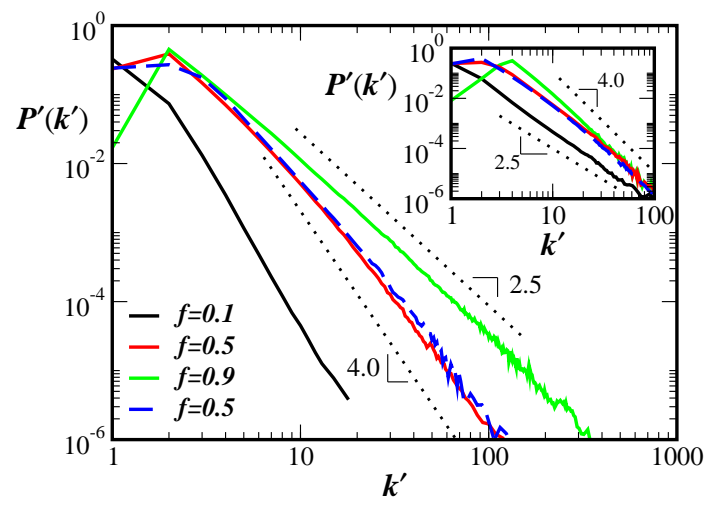

FIG. 2: Degree distribution of the depreciated network. This result was obtained for 10 network realizations of size $N_{v}=10^{5}$. For each network realization, the depreciation has been applied 10 times. In the main panel we show the degree distribution for networks with $\gamma=2.5$ submitted to CB failure with $\alpha=0.5$. From Eq. (1) we expect the value $\gamma^{\prime}=4.0$ for the depreciated network. As a guide to the eye, the dotted lines indicate the power-law decays with exponents 2.5 and 4 . One can see that for small degree the distribution initially decays with a slope very close to $\gamma$, and then crosses over to a decay with a slope close to $\gamma^{\prime}$ at a scale that depends on the fraction $f$ of edges in the depreciated network. This shows that the topology-dependent failure process introduces a characteristic scale in the degree distribution of the originally scale-free network. In the inset we show results for networks with $\gamma=4$ subjected to PB failure with $\alpha=-1$, which is equivalent to an exponent $\gamma^{\prime}=2.5$. Contrary to the result of the main panel, one sees a crossover from a slope $\gamma^{\prime}$ at small degree to a slope $\gamma$ at large degree. The minimum degree was set to $k_{\text {min }}=4$ in the results of the inset and $k_{\min }=2$ in the main panel. Surprisingly, for the case $f=0.5$ the degree distributions in the inset and the main panel are remarkably similar. To illustrate this similarity we included the results for $f=0.5$ from the main panel in the inset and vice versa; these are the dashed (blue) lines. Although the two cases start with distinct degree distributions at $f=1$, we obtain similar distributions at a certain point of the depreciation process.

fragile network regime. On the other hand, when $\gamma<3$, one obtains $\left\langle k^{\prime 2}\right\rangle \propto k_{\text {max }}^{3-\gamma}$, where $k_{\max }$ is the largest degree of a finite network. If no other constraint is imposed, $k_{\text {max }} \propto N_{v}^{1 /(\gamma-1)}$ [2, 14], resulting in $f_{c} \propto N_{v}^{-(3-\gamma) /(\gamma-1)}$, for $2<\gamma<3$. As long as $\gamma<3, f_{c} \rightarrow 0$ as $N_{v} \rightarrow \infty$, characterizing the robust network regime.

For CB $(\alpha>0)$ it is possible that $\gamma \leq 3$ while $\gamma^{\prime}>3$. Since the tail of the distribution at large values of $k^{\prime}$ decays as $P^{\prime}\left(k^{\prime}\right) \propto k^{\prime-\gamma^{\prime}}$, the second moment $\left\langle k^{\prime 2}\right\rangle$ no longer diverges and a robust network becomes fragile under CB. On the other hand, for PB $(\alpha<0), \gamma^{\prime}<\gamma$, and the larger exponent, $\gamma$, should control the decay of the tail of the degree distribution. Therefore, one may think that for $\gamma>3,\left\langle k^{\prime 2}\right\rangle$ also does not diverge under $\mathrm{PB}$ and that a fragile network cannot turn robust. This simple reasoning is mistaken, however, as we show in the following.
It is possible that the crossover scale $k_{s}$ becomes so large that in practice it does not influence a finite network. That is the case, for instance, of the distribution for $f=0.1$ and $N_{v}=10^{5}$, shown in the inset of Fig. 2 . It may be the case that, at the critical point of PB failure, a network with $\gamma^{\prime} \leq 3$ never displays an observable crossover, irrespective of the system size, that is,

$$
k_{s}\left(f_{c}\left(N_{v}\right)\right)>k_{\max }\left(N_{v}\right) .
$$

When Eq. (5) holds true, Eq. (41) may be rewritten as $k^{\prime}=$ $C\left(f_{c}\right) k^{1-\alpha}$. In this limit one can find a linear relation between the parameter $C\left(f_{c}\right)$ and the occupation fraction $f_{c}=\left\langle k^{\prime}\right\rangle /\langle k\rangle=C\left\langle k^{1-\alpha}\right\rangle /\langle k\rangle$. The second moment can be identified as $\left\langle k^{\prime 2}\right\rangle=C^{2}\left(\left\langle k^{2-2 \alpha}\right\rangle-\left\langle k^{1-2 \alpha}\right\rangle\right)+C\left\langle k^{1-\alpha}\right\rangle$. From the critical condition $\left\langle k^{\prime 2}\right\rangle /\left\langle k^{\prime}\right\rangle>2$ we get

$$
f_{c}=\frac{\left\langle k^{1-\alpha}\right\rangle^{2}}{\langle k\rangle\left[\left\langle k^{2-2 \alpha}\right\rangle-\left\langle k^{1-2 \alpha}\right\rangle\right]} .
$$

As long as $2<\gamma^{\prime}$ and $2<\gamma$, the moments $\left\langle k^{1-\alpha}\right\rangle$ and $\langle k\rangle$ should both converge to finite values independent of $N_{v}$. The moment $\left\langle k^{1-2 \alpha}\right\rangle$ may or may not diverge, but at large scales it will grow slower than $\left\langle k^{2-2 \alpha}\right\rangle \rightarrow k_{\max }^{3-2 \alpha-\gamma}$. Thus, considering that $k_{\max } \propto N_{v}^{1 /(\gamma-1)}$ [2], we arrive at the behavior

$$
f_{c} \propto N_{v}^{\frac{\gamma^{\prime}-3}{\gamma^{\prime}-1}}
$$

This result shows not only that PB can turn a fragile network robust but also that the critical exponent with which the threshold $f_{c}$ approaches zero is the same as expected for normal percolation $(\alpha=0)$ for a network with a degree distribution decaying as $P(k) \propto k^{-\gamma^{\prime}}$.

We can now check the self-consistency of our initial assumption, Eq. (5), that networks with $\gamma^{\prime} \leq 3$ at the critical point of $\mathrm{PB}$ failure do not present a crossover. As mentioned before, the crossover scale is given by $k_{s} \propto$ $C(f)^{1 / \alpha}$. At the critical point Eq. (7) then implies $k_{s} \propto$ $N_{v}^{\left(\gamma^{\prime}-3\right) /\left[\alpha\left(\gamma^{\prime}-1\right)\right]}$, while $k_{\max } \propto N_{v}^{1 /(\gamma-1)}$. From this we obtain $\left(k_{s} / k_{\max }\right)^{-\alpha(\gamma-1)} \propto N_{v}^{3-\gamma-\alpha}$. As long as $\alpha<$ $3-\gamma$, the crossover scale grows faster than the maximum degree, implying that critical networks with $\gamma^{\prime} \leq 3$ and sufficiently strong $\mathrm{PB}$ do not display a crossover in their degree distributions. However, for weak PB, $3-\gamma<\alpha<$ 0, Eq. (5) is violated and the network may remain fragile.

Figure 3 shows numerical results confirming that a robust network with $\gamma=2.5$ submitted to CB failure with $\alpha=0.5$ turns fragile. In contrast, the second set of results demonstrates that a fragile network turns robust even for $\alpha=3-\gamma=-1$, which is on the borderline between weak and strong PB.

Our assumption that the probability of failure depends on degree $k$ can be justified in different contexts. In artificial networks, e.g., air transportation [15], the capacity of the nodes scales with $k$. Depending on whether $k$ or capacity grows faster, this system should be better modelled by $\mathrm{CP}$ or PB failure, respectively. Software systems [16] and metabolic networks [17] consist of 


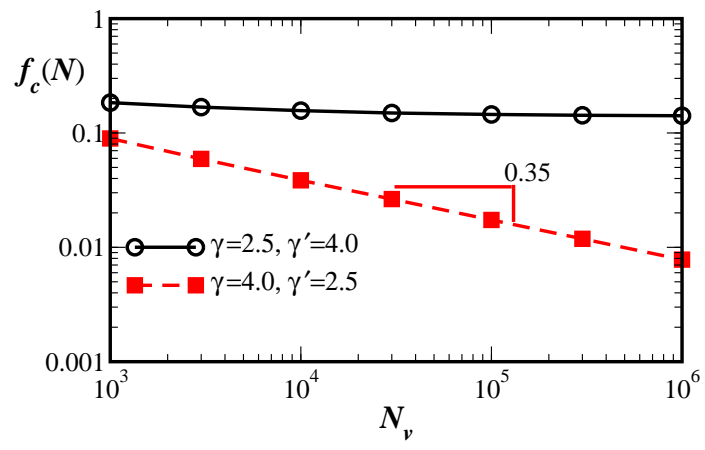

FIG. 3: The values $f_{c}$ of the fraction of edges in the depreciated network at the critical condition as a function of the network size $N_{v}$. Criticality is defined as the point where $\left\langle k^{\prime 2}\right\rangle /\left\langle k^{\prime}\right\rangle=2$ [2], To compute this critical fraction we average over $10^{4}$ network realizations for each set of parameters. For each network we apply the percolation processes 100 times. For networks with $\gamma=2.5$ submitted to $\mathrm{CB}$ with an effective value $\gamma^{\prime}=4$ (continuous black line), we observe that the critical fraction $f_{c}$ converges to a finite value as $N_{v}$ grows, confirming the conjecture that a robust network may turn fragile under CB. The opposite case, a network with $\gamma=4$ submitted to $\mathrm{PB}$ with an effective $\gamma^{\prime}=2.5$ (dashed red line), has a critical fraction that decays with $N_{v}$ as a power law, $f_{c} \propto N_{v}^{-1 / \nu}$. The best fit to the data in this case results in $1 / \nu=0.35 \pm 0.02$, consistent with the value $1 / 3$ expected from Eq. (7). This result shows that a fragile network under $\mathrm{PB}$ can behave in the same fashion as a robust network with a degree distribution controlled by $\gamma^{\prime}$ under random failure.

many agents/nodes acting together in some function. If all agents are needed, the lack of any of them can interrupt the process. Alternatively, if any of the agents can start it, only removal of all edges halts the process. In both cases, depending on $k$, the edges turn more fragile or robust. Further, if disrupting the network is desirable, as in gene fusion networks of cancer development [18] or terrorist networks, the design of a dynamical process that targets links between the most connected nodes (CB) would be more efficient to globally break down the system. Also, to reduce the risk of epidemic spreading, it is better to disinfect/immunize connections between hubs than connections between small (air-)ports. Note that our analysis does not account for dynamical correlations in the failure process. It can happen that removal of a single edge triggers a breakdown, even if this edge only links to one of the least connected nodes [19].

We conclude that topologically biased failure can have a dramatic effect on the percolation properties of scalefree networks. For central bias ( $\mathrm{CB}, 0<\alpha<1$ ), the degree distribution initially decays with the exponent $\gamma$ up to a certain scale that depends on the fraction of occupied edges, and then crosses over to a decay with an exponent $\gamma^{\prime}>\gamma$ defined as in Eq. (1). For peripheral bias (PB, $2-\gamma<\alpha<0)$ the crossover is also present but with $\gamma^{\prime}$ controlling the early decay and the exponent $\gamma>\gamma^{\prime}$ appearing at large degree. Our results also demonstrate that a robust network, for which the critical fraction $f_{c}$ converges to zero as the network grows, may turn fragile when subjected to $\mathrm{CB}(\alpha>0)$. Conversely, a fragile network, for which the critical point is larger than zero at any system size, may become robust when subjected to strong PB, $\alpha<3-\gamma$. Fragility or robustness of a network is thus not only dependent on the exponent $\gamma$ but can be tuned quantitatively by the exponent $\alpha$ characterizing the topological bias.

We thank Hans Hooyberghs for discussions, and FWOVlaanderen Project G.0222.02, CCSS, CNPq, CAPES, FUNCAP, and FINEP for financial support.
[1] R. Albert, H. Jeong, and A.-L. Barabási, Nature 406 , 378 (2000).

[2] R. Cohen, K. Erez, D. ben-Avraham, and S. Havlin, Phys. Rev. Lett. 85, 4626 (2000)

[3] Y. I. Wolf, G. Karev, and E. V. Koonin, Bioessays 24, 105 (2002).

[4] R. Cohen, K. Erez, D. ben-Avraham, and S. Havlin, Phys. Rev. Lett. 86, 3682 (2001).

[5] A. V. Goltsev, S. N. Dorogovtsev, and J. F. F. Mendes, Phys. Rev. E 67, 026123 (2003).

[6] C. V. Giuraniuc, J. P. L. Hatchett, J. O. Indekeu, M. Leone, I. Pérez Castillo, B. Van Schaeybroeck, and C. Vanderzande, Phys. Rev. Lett. 95, 098701 (2005).

[7] C. V. Giuraniuc, J. P. L. Hatchett, J. O. Indekeu, M. Leone, I. Pérez Castillo, B. Van Schaeybroeck, and C. Vanderzande, Phys. Rev. E 74, 036108 (2006).

[8] L. K. Gallos, R. Cohen, P. Argyrakis, A. Bunde, and S. Havlin, Phys. Rev. Lett. 94, 188701 (2005).

[9] Z. Wu, L. A. Braunstein, V. Colizza, R. Cohen, S. Havlin, and H. E. Stanley, Phys. Rev. E 74, 056104 (2006).
[10] D. S. Callaway, M. E. J. Newman, S. H. Strogatz, and D. J. Watts, Phys. Rev. Lett. 85, 5468 (2000).

[11] M. E. J. Newman, S. H. Strogatz, and D. J. Watts, Phys. Rev. E 64, 026118 (2001).

[12] R. Cohen, K. Erez, D. ben-Avraham, and S. Havlin, Phys. Rev. E 66, 036113 (2002).

[13] C. M. Fortuin and P. W. Kasteleyn, Physica 57, 536 (1972).

[14] A. A. Moreira, J. S. Andrade, L. A. N. Amaral, Phys. Rev. Lett. 89, 268703 (2002).

[15] R. Guimer, S. Mossa, A. Turtschi, and L. A. N. Amaral, Proc. Nat. Acad. Sci. USA 102, 7794 (2005).

[16] C. R. Myers,Phys. Rev. E 68, 046116 (2003).

[17] R. Sharan and T. Ideker, Nature Biotechnology 24, 427 (2006).

[18] M. Hglund, A. Frigyesi, and F. Mitelman, Oncogene 25, 2674 (2006).

[19] A. Samal, S. Singh, V. Giri, S. Krishna, N. Raghuram, S.Jain, BMC Bioinformatics 7:118 (2006). 